\title{
Vocabulário controlado para arquivos universitários: a experiência do VTArq-UNESP
}

Vocabulario controlado para archivos universitarios: la experiencia del VTArq de UNESP (Brasil)

An archival controlled vocabulary for university archives: the experience of VTArq (UNESP, Brazil)

\section{Walter MOREIRA, Mariângela Spotti Lopes FUJITA, Luciana Beatriz Piovezan dos SANTOS}

Universidade Estadual Paulista, Av. Hygino Muzzi Filho, 737, Campus Universitário, CEP. 17525900, Marília, SP, Brasil; \{ walter.moreira | mariangelaslf57 | lbpiovezan \}@gmail.com

\section{Resumen}

Se presenta la construcción de un vocabulario controlado común para a las tareas de procesamiento de información del archivo de la Universidade Estadual Paulista (VTArq-UNESP). Se consultó con las unidades de archivo de la universidad para la identificación y recopilación de expresiones utilizadas con eficacia en la representación de la documentación. Seguidamente, se investigó la adecuación del vocabulario con programas informáticos de gestión terminológica. La experiencia demostró la necesidad de un vocabulario específico para los archivos de la universidad y de su normalización para la organización y recuperación de información.

Palabras clave: Tesauros. Planificación. Construcción. Archivística. Gestión de documentos. Archivos universitarios.

\section{Introdução}

$\mathrm{Na}$ área da arquivologia, as discussões sobre aspectos teóricos e metodológicos envolvidos na construção e na aplicação de instrumentos de controle vocabular, denominados genericamente pela expressão "vocabulários controlados", ainda não são realizadas com a frequência e o aprofundamento com que são encontrados na biblioteconomia e na documentação, disciplinas citadas para fins de comparação (Davanzo; Moreira, 2015; Moreira; Davanzo; Moraes, 2015). Mesmo a terminologia arquivística referente a esses instrumentos de organização e representação do conhecimento ainda é incipiente.

Dentre algumas experiências bem-sucedidas, pode-se destacar o trabalho do Grupo de Archiveros Municipales de Madrid, em atividade desde 1981, formado por profissionais de arquivo e de gestão documental municipal da Comunidade de Madri. Esse Grupo organiza, desde 1982 as Jornadas de Archivos Municipales. Na sua edição de número dezoito, realizada em 2010, o Grupo publicou, como resultado de uma comissão especialmente criada para este fim, o Tesauro de archivos municipales (Grupo de Archiveros de Madrid, 2010), trabalho iniciado em 1997. Ainda

\begin{abstract}
The experience of construction of a controlled vocabulary for processing the university records of the Universidade Estadual Paulista (UNESP VTArq) is presented. First, the different units of the university were consulted for the identification and collection of the expressions effectively used for representing the documentation. Thereafter, the adequacy of the vocabulary was assessed with the help of terminology management software. The experience shows the need of vocabulary specificity and standardization to support the organization and retrieval of information in the field of university archives.
\end{abstract}

Keywords: Thesauri. Planning. Construction. Archival science. Record management. University archives.

que tal instrumento seja destinado a documentação distinta da que se ocupa o trabalho que ora se apresenta, a experiência relatada e, principalmente, o detalhamento da metodologia, revestem-se de substancial interesse para o projeto que se apresenta ainda em desenvolvimento.

Em vista do volume de informações e de documentos em circulação e da impossibilidade de gerenciá-los em termos de suas representações documentárias sem o recurso de instrumentos adequados, a construção e a utilização de vocabulários controlados tornou-se necessária em quaisquer unidades de informação, incluindo-se, vale a redundância, as unidades de informação arquivística.

Nesse contexto, a efetiva compreensão da complexidade do fluxo documentário e a busca por soluções eficazes para os problemas de representação e recuperação da informação arquivística atuam como fatores motivadores para o investimento que a Universidade Estadual Paulista (UNESP) vem realizando na construção de um vocabulário controlado que possa ser utilizado amplamente pelas diversas unidades que a compõem bem como pelos demais arquivos universitários com o objetivo de compartilhamento 
de experiências teóricas e metodológicas e, sobretudo, no aprimoramento do controle de vocabulário arquivístico.

\section{Vocabulário controlado como instrumento de organização e representação do conhecimento}

A organização do conhecimento preocupa-se com os aspectos teóricos e metodológicos referentes ao modo pelo qual o conhecimento será classificado ou estruturado nas diversas áreas. Observando-se este seu caráter aplicado, notadamente na ciência da informação, é mister entrelaçar o conceito de organização do conhecimento com os conceitos de representação do conhecimento e, inclusive, com os aspectos que envolvem a recuperação da informação. A rigor, respeitando-se essa faceta referente à aplicação, criam-se sistemas de organização para que, por seu intermédio, sejam possíveis a organização e a recuperação da informação.

García Marco (1993, p. 100, tradução livre) reconhece o caráter científico-aplicado da organização do conhecimento quando informa que seu objetivo é "melhorar a circulação da informação dentro e por meio de sistemas mediadores - os centros de seleção, armazenamento, recuperação e disseminação de informação - para produzir novo conhecimento e/ou facilitar o acesso ao [conhecimento] existente".

A temática que ocupa este trabalho não diz respeito, contudo, aos problemas diretamente envolvidos na recuperação da informação, apenas os aborda de modo indireto, com base na compreensão sistêmica das inter-relações apresentadas brevemente no parágrafo anterior. Trata-se mais especificamente nesse trabalho da organização e representação do conhecimento, uma disciplina oriunda da confluência da ciência da informação com as ciências cognitivas, a linguística, a terminologia, a ciência da computação e a lógica.

A institucionalização e a legitimação social e profissional da organização e representação do conhecimento ocorrem também por meio do trinômio desenvolvimento, utilização e aprimoramento dos sistemas de organização do conhecimento (SOC, ou KOS, knowledge organization systems, na terminologia mais universalizada adotada na língua inglesa). Dentre os SOC mais conhecidos incluem-se os sistemas de classificação, os tesauros, as taxonomias, as ontologias e os topic maps. Considerando-se apenas as características que os aproximam, apresentam elementos que podem ser subsumidos na expressão genérica "vocabulários controlados". Note-se bem que não se está afirmando que o controle vocabular é a finalidade ou a função primeira de tais instrumentos, mas sim que todos eles manifestam em algum momento, em maior ou menor grau, algum nível de padronização ou de normalização terminológica no processo.

O nível demandado de controle do vocabulário irá variar, naturalmente, conforme a configuração estrutural do SOC e de acordo com o contexto e os objetivos de seu desenvolvimento, bem como pelo caráter de sua aplicação. Exige-se, por exemplo, maior precisão terminológica num tesauro do que num sistema de classificação, do mesmo modo que se espera de uma ontologia um uso do léxico mais acurado do que aquele revelado por uma taxonomia.

Os vocabulários controlados desempenham um importante papel na redução dos eventuais ruídos presentes na comunicação entre os sistemas de informação documentária e seus usuários. Desse modo atuam como instrumentos auxiliares indispensáveis aos processos de organização, representação e recuperação da informação.

Além de aumentar a eficácia do sistema nas ações de representação da informação, um vocabulário controlado eficaz também amplia, em razão diretamente proporcional, sua confiabilidade. A noção de representação, neste caso, refere-se à representação como processo, traduzido no ato que realizam, de modo assíncrono, o profissional indexador e o usuário. Considerando-se que ambos se interessam pela localização e pela utilização efetiva da informação, ou seja, concorrem para o mesmo fim, a linguagem natural torna-se um obstáculo quando se revela seu caráter naturalmente polissêmico. Assim, o instrumento de controle vocabular funciona como instrumento de tradução, mediador entre as expressões que representam conceitos formulados pelos usuários e pelos profissionais indexadores. O vocabulário controlado permite traduzir a linguagem do usuário e a linguagem do sistema para uma linguagem documentária que possa atuar como língua franca nas situações em que se requer sua interlocução.

A partir dessa função intermediadora, atribui-se também aos vocabulários controlados uma função de protagonismo social na disponibilização e na apropriação da informação, bem como na promoção do conhecimento como dínamo social (Barité, 2001). O compartilhamento social da informação é, afinal, devedor dos modelos de organização e representação da informação e do conhecimento. 


\section{A proposta do VTArq-UNESP}

A UNESP é uma instituição de ensino superior relativamente jovem, completando 40 anos em 2016, que visa a oferecer ensino superior público, gratuito e de qualidade. Está geograficamente localizada de forma descentralizada no estado de São Paulo, no Brasil: são 34 unidades distribuídas em 24 cidades.

Seria utopia imaginar que a cultura organizacional que imprime identidade à universidade, a qualquer universidade, e a identificação e descrição de seu fluxo documentário possam atuar como garantia para a coerência na representação dos seus documentos. Essas dificuldades, no caso da UNESP, acentuam-se em vista do caráter de descentralidade, e até mesmo de certa incomunicação provocada, entre outros, pela barreira geográfica a qual cria, muitas vezes, condições de isolamento.

Nesse contexto, visando a garantir a eficácia necessária ao bom fluxo da informação arquivística, tornou-se imperativo investir na construção de um vocabulário controlado comum que pudesse subsidiar todas as unidades em suas tarefas de tratamento da informação arquivística nas diferentes seções de comunicação que compõem as unidades de ensino.

A construção do referido vocabulário controlado foi iniciada em resposta à necessidade detectada de forma mais premente a partir da implantação do Sistema de Gestão Eletrônica de Documentos (SIGAD). Dentre as novidades e desafios que o SIGAD apresentou, estava a utilização de um campo destinado à inserção de palavras-chave. Bem poucas unidades já utilizavam, no sistema antigo, o recurso das palavras-chave, e esse recurso era realizado sem qualquer elemento de controle de vocabulário.

Para a construção do Vocabulário de Termos Arquivísticos da UNESP (VTArq- UNESP) foram consultadas as seções técnicas de comunicação dos diversos campi, visando à identificação e compilação das expressões efetivamente utilizadas na representação da documentação que circulava nas seções técnicas de comunicação. Completada essa fase, realizou-se pesquisa terminológica visando à identificação e padronização dos termos candidatos, distinguindo-os entre termos preferidos e termos não preferidos. Os procedimentos metodológicos utilizados na condução da pesquisa terminológica estão descritos no capítulo 3 deste trabalho.

A subcomissão de atualização e manutenção do VTArq-UNESP iniciou seus trabalhos em março de 2014. Vincula-se hierarquicamente à Comissão de Avaliação de Documentos e Acesso (CADA), da mesma universidade.

O VTArq-UNESP foi construído em função de solicitação do Grupo Gestor do Sistema de Gestão Documental Eletrônica (SIGAD) à CADA.

CADA é a atual denominação da comissão responsável pela avaliação dos documentos de arquivos conforme o Decreto no 58.052, de 16 de maio de 2012, que regulamenta, no estado de São Paulo, a Lei de Acesso à Informação - Lei Federal no 12.527, de 18 de novembro de 2011. O Decreto também criou em todos os órgãos e entidades de administração pública estadual os Serviços de Informação ao Cidadão (SIC), os quais são responsáveis pelo atendimento presencial ou eletrônico ao cidadão e pelo encaminhamento das solicitações aos SICs setoriais.

Na UNESP, a instalação do SIC, em 2012, ocorreu de forma centralizada junto ao Gabinete do Reitor, mas organizada mediante a determinação de que as seções técnicas de comunicação de cada unidade universitária ou unidade experimental, onde se localizam os arquivos correntes, se tornassem unidades locais do SIC central, funcionando como um sistema de arquivos correntes para provimento de qualquer solicitação de informação. Dessa forma, a gestão documentária é necessária nos arquivos universitários porque são, no entendimento da CADA, o local de destinação e organização da documentação produzida pelas atividades meio e fins da UNESP.

O SIGAD é um sistema que instrumentaliza e dá visibilidade à gestão documental das seções técnicas de comunicação da UNESP. Para seu completo funcionamento necessita dos instrumentos de organização e representação da informação da área arquivística que são o plano de classificação de atividades meio e o plano de classificação de atividades fins, com tabela de temporalidade compatíveis com o Plano de Classificação e Tabela de Temporalidade do Sistema de Arquivos do Estado de São Paulo (SAESP), o qual coordena o sistema SIC do estado de São Paulo.

O VTArq-UNESP foi elaborado não em função do Decreto Lei Estadual, que não prevề este instrumento de organização e representação da informação, mas por solicitação das seções técnicas de comunicação, que tinham uma prática comum de fazer a determinação de assuntos em todos os denominados processos que organizam os documentos de uma mesma procedência organizacional.

Assim, a origem do VTArq-UNESP está na compatibilização de três listas alfabéticas de "palavras-chave" compiladas por seções técnicas de 
comunicação da UNESP. Essa compilação, sem método ou técnica de elaboração de vocabulários ou mesmo de recursos de pesquisa terminológica, foi realizada ao longo do tempo de prática dessas seções, o que determinou, em alguns casos, a autorização desses termos sob garantia de uso

Para a necessária normalização terminológica das listas alfabéticas oriundas das seções de comunicação desempenhou-se procedimentos de controle de vocabulário a partir das diretrizes estabelecidas pela UNESCO (1993) e com o uso de um modelo de ficha terminológica adaptado aos interesses e particularidades do projeto. A adaptação da ficha terminológica (Quadro I) deu-se com base nos modelos propostos por Cervantes (2004), Pavel e Nolet (2002) e Barros (2004).

\begin{tabular}{|c|c|}
\hline \multicolumn{2}{|l|}{ Termo: } \\
\hline $\begin{array}{l}\text { Categoria gra- } \\
\text { matical: }\end{array}$ & Sigla/Acrônimo: \\
\hline \multicolumn{2}{|c|}{$\begin{array}{l}\text { Unidade UNESP: [Unidade na qual o termo é utili- } \\
\text { zado, conforme lista fornecida pelas seções técni- } \\
\text { cas de comunicação] }\end{array}$} \\
\hline \multicolumn{2}{|c|}{ Outras designações } \\
\hline \multicolumn{2}{|c|}{ Contexto de ocorrência } \\
\hline \multicolumn{2}{|c|}{ Referência completa } \\
\hline $\begin{array}{l}\text { Conceito insti- } \\
\text { tucional }\end{array}$ & $\begin{array}{l}\text { [Observação da ocorrência do } \\
\text { termo/conceito em documentos } \\
\text { legais da UNESP] }\end{array}$ \\
\hline \multicolumn{2}{|c|}{ Referência Completa } \\
\hline $\begin{array}{l}\text { Compreensão } \\
\text { do servidor }\end{array}$ & $\begin{array}{l}\text { [Informação obtida junto às } \\
\text { seções técnicas de comunicação] }\end{array}$ \\
\hline $\begin{array}{l}\text { Responsável } \\
\text { pela coleta: }\end{array}$ & Data: \\
\hline
\end{tabular}

Quadro I. Ficha terminológica de trabalho (adaptado de Cervantes, 2004; Pavel e Nolet, 2002; e Barros, 2004)

O uso da ficha terminológica teve como objetivo principal, na primeira fase, o controle de sinonímia, de número e gênero e de forma gramatical, além do fornecimento de elementos para a identificação de relações de equivalência entre os termos. Um elemento que se revelou particularmente importante na ficha terminológica utilizada foram os campos denominados "conceito institucional", referindo-se à representação da linguagem técnica da UNESP, por meio da terminologia empregada na documentação oficial da universidade, e "compreensão do servidor", referindo-se ao contexto de uso pela comunidade de servidores e docentes da UNESP.
O termo "água", por exemplo, que aparecia na lista de palavras autorizadas de duas seções técnicas de comunicação e que parece vago por seu amplo grau de generalidade, pôde ser substituído, após a identificação de seu uso efetivo (contexto de uso) pelo termo "conta de água".

De outro lado, as sutilezas do sentido e das relações semânticas entre termos "admissão" (servidor admitido pelo regime autárquico), "contratação" (servidor admitido pelo regime da Consolidação das Leis do Trabalho - CLT) e "nomeação" (servidor admitido pelo regime efetivo) só puderam ser devidamente captadas com o apoio da legislação específica aplicada na UNESP.

Atualmente, o VTArq-UNESP está em processo final de adequação à terminologia dos Planos de Classificação da UNESP para sua disponibilização final dentro do SIGAD. Nessa fase, também estão sendo realizados os testes de fluxo navegacional do vocabulário no ambiente do software. Essa revisão e os testes precedem, necessariamente, o uso, uma vez que sua terminologia precisa estar adequada à produção documental estadual e universitária determinantes da procedência arquivística. Desse modo, a liberação para o uso do vocabulário somente será possível após completadas as fases de revisão, adequação e testes. Posteriormente a essa etapa o corpo técnico responsável pelas seções técnicas de comunicação serão devidamente informados e capacitados para a utilização efetiva do VTArq-UNESP.

Com a finalização do plano de classificação de atividades meio e tabela de temporalidade da UNESP (Troitiño, 2016) foi possível realizar uma adequação com base no vocabulário empregado. Aguarda-se a finalização do plano de classificação de atividades fins para uma segunda adequação de vocabulário.

A elaboração do VTArq-UNESP, tem origem nas listas alfabéticas como primeira etapa que se constituiu da normalização e estruturação logica e semântica dos termos compilados com uso do software TemaTres, versão 1.6. Na sequencia, a segunda etapa consistiu da adequação de vocabulário ao Plano de classificação de atividades meio da UNESP.

O TemaTres é uma aplicação web utilizada para a gestão de representações linguísticas formais do conhecimento, incluindo vocabulários controlados, taxonomias, tesauros e listas de cabeçalhos de assunto (Gonzales Aguilar, Ramírez Posada, Ferreyra, 2012; Ferreyra, 2016).

Além de ser um software reconhecido pela comunidade científica (Scornik; Sosa, 2007; Martínez- 
González; Alvite-Díez, 2014;), a escolha do TemaTres se deu também em função de sua gratuidade e por conta dos recursos de promoção de interoperabilidade que a ferramenta oferece, como, por exemplo, a exportação da estrutura do vocabulário controlado em formato Simple Knowledge Organization System (SKOS).

O SKOS é uma linguagem formal baseada em Resource Description Framework (RDF) que pode ser utilizada para a construção de representações documentárias que incluem os tesauros e os sistemas de classificação (World Wide Web Consortium - W3C, 2012). Trata-se de um esforço de promoção de interoperabilidade baseado na concepção de que os sistemas de organização do conhecimento guardam muitas semelhanças, tanto em relação ao modo como são estruturados quanto às suas aplicações. A identificação dessas similaridades e sua explicitação em linguagem formal compreensível por máquina facilita as ações de interoperablidade na construção e uso de vocabulários controlados.

\section{Observações sobre os resultados do projeto}

O VTArq-UNESP possui, após as primeiras revisões, um total de 1.249 termos, sendo 1.087 termos preferidos e 162 termos não preferidos. Por se tratar de um projeto que envolve investigação e execução em médio prazo, os resultados apresentados são parciais. Verificou-se que será necessário, em continuidade, avaliar o uso do VTArq-UNESP no atendimento às necessidades da produção e gestão documental dos arquivos correntes da UNESP no Sistema SIGAD.

A composição atual do VTArq tem a seguinte origem: a) as listas de expressões utilizadas pelas três seções técnicas de comunicação que foram apresentadas aos pesquisadores responsáveis pelo projeto e b) o Plano de Classificação de Atividades Meio da UNESP.

Para composição do conjunto de termos preferidos e não-preferidos, foi realizada a análise terminológica de todos os termos candidatos, isto é, todas as entradas resultantes dos instrumentos citados no parágrafo anterior. Essa decisão foi tomada em observação às garantias necessárias ao funcionamento do vocabulário controlado. Refere-se, especificamente, à garantia de uso (pela incorporação, quando possível, das expressões efetivamente utilizadas pelos profissionais responsáveis nas seções técnicas de comunicação) e à garantia institucional (pelo respeito ao plano de classificação instituído).
Considerando-se as diferentes naturezas e propósitos dos instrumentos, a tarefa de compatibilização terminológica entre o vocabulário controlado e o plano de classificação revelou-se mais complexa do que se poderia inicialmente crer. $\mathrm{O}$ recurso utilizado para a compatibilização foi o cotejo direto entre os termos presentes nos dois instrumentos buscando-se identificar sinônimos e quase-sinônimos e verificar quais as entradas elencadas no plano de classificação que poderiam ser utilizadas na construção de novos termos preferidos ou não preferidos no VTArqUNESP.

Com vistas a organizar hierarquicamente os termos foram utilizadas como proposta de macrocategorização as categorias definidas pelo Classification Research Group (CRG), conforme sua apresentação em Frické (2012, p. 214). Em tradução livre, são as seguintes: coisa, tipo, parte (órgão, constituinte), propriedade, material, processo (uma ação interna ao item), operação (uma ação realizada no item), paciente (objeto da ação, matéria-prima), produto (substância), subproduto, agente, espaço, tempo.

Para o desenvolvimento pleno do projeto e considerando-se principalmente a perspectiva de hibridez nos espaços de informação, há que se observar diretrizes e normas internacionais aplicadas tanto à descrição arquivística quanto à elaboração e manutenção de tesauros (Davanzo, 2016). Neste aspecto, destacam-se, sem prejuízo de outras correlacionadas, as seguintes normas ISAAR(CPF): norma internacional de registro de autoridade arquivística para entidades coletivas, pessoas e famílias (Conselho Internacional de Arquivos, 2004); ISO 25964: information and documentation: thesauri and interoperability with other vocabularies (International Organization for Standardization, 2011a; 2011b) e as Guidelines for Authority Records and References (International Federation of Library Associations and Institutions, 2001).

Em sequência, está prevista a capacitação de todos os responsáveis pelas seções técnicas de comunicação na UNESP para a função e uso do VTArq-UNESP. Nessa oportunidade, será estimulada a criação de um grupo de atualização e manutenção do Vocabulário, que deverá ser permanente e acompanhado pelo grupo responsável pela elaboração.

O Grupo de elaboração será responsável por realizar o monitoramento avaliativo de uso do VTArq-UNESP, com metodologia de avaliação extrínseca e intrínseca aplicadas anualmente além da verificação quantitativa e qualitativa dos termos e sua utilização. 


\section{Considerações finais}

Diante do exposto consideramos relevante reafirmar a importância do debate relativo aos instrumentos de controle de vocabulário, seus aspectos teórico-metodológicos e sua aplicação, direcionado às unidades de informação arquivística visando ao desenvolvimento das práticas de organização e recuperação da informação em sua responsabilidade.

No contexto das seções técnicas de comunicação das unidades universitárias da UNESP, com o investimento na construção deste instrumento normalizado de representação da informação espera-se não apenas atender aos dispositivos legais, mas também aperfeiçoar a própria gestão documental ali realizada, vindo a beneficiar a comunidade acadêmica.

\section{Referências}

Barite, Mario (2001). Organización del conocimiento: um nuevo marco teórico-conceptual em Bibliotecología y Documentación. // Carrara, Kester (Org.). Educação, universidade e pesquisa. Marília: Unesp-Marília, 2001. 35-60.

Barros, Lidia Almeida (2004). Curso básico de Terminologia. São Paulo: Edusp, 2004.

Cervantes, Brígida Maria Nogueira (2004). Contribuição para a terminologia do processo de inteligência competitiva: estudo teórico e metodológico. 2004. 209f. Dissertação (Mestrado). Faculdade de Filosofia e Ciências, Universidade Estadual Paulista, Marília, 2004.

Conselho Internacional de arquivos (2004). ISAAR(CPF): norma internacional de registro de autoridade arquivística para entidades coletivas, pessoas e famílias/tradução de Vitor Manoel Marques da Fonseca. Rio de Janeiro: Arquivo Nacional, 2004.

Davanzo, Luciana; Moreira, Walter (2015). A ISAAR(CPF) e os vocabulários controlados: uma aproximação preliminar. // Zabala Vázquez, J.; Sánchez Jiménez, R.; García Moreno, M.A. (Orgs.). Desafíos y oportunidades de las Ciencias de la Información y la Documentación en la era digital: actas del VII Encuentro Ibérico EDICIC 2015 (Madrid, 16 y 17 de noviembre de 2015). Madrid: Universidad Complutense de Madrid, 2015.

Ferreyra, Diego (2016). TemaTres. http://www.vocabularyser ver.com/index.html (2016-03-16).

Frické, Martin (2012). Logic and the organization of information. New York: Springer, 2012.

García-Marco, Francisco Javier (1993). Paradigmas científicos en representación y recuperación de la información. // García-Marco, Francisco Javier (Ed.). Organización del conocimiento en sistemas de información y documentación: actas del I Encuentro de ISKO-España, Madri, 4 y 5 nov. 1993. Zaragoza: Universidad de Zaragoza, 1993.

Gonzales Aguilar, Audilio; Ramírez Posada, María; Ferreyra, Diego (2012). Tematres: software para gestionar tesauros. // El profesional de la información. 21:3 (MayoJunio) 319-325.

Grupo de Archiveros Muncipales de Madrid (2010). XVII Jornadas de Archivos Municipales: "Pilares de la eadministración: cuadro de clasificación y tesauro": Tesauro de Archivos Municipales. San Sebastián de los Reys: B.O.C.M., 2010.

International Federation of Library Associations and Institutions (2001). Guidelines for Authority Records and References. 2.ed. Munchen: K.G. Saur, 2001.

International Organization for Standardization (2011a). ISO 25964: information and documentation: thesauri and interoperability with other vocabularies - part 1: thesauri for information retrieval. Genebra, 2011.

International Organization for Standardization (2011b). ISO 25964: information and documentation- thesauri and interoperability with other vocabularies - part 2: interoperability with other vocabularies. Genebra, 2011.

Martínez-González, M. Mercedes; Alvite-Díez, María-Luisa (2014). On the evaluation of thesaurus tools compatible with the Semantic Web. // Journal of Information Science. 40:6 711-722.

Moreira, Walter; Davanzo, Luciana; Moraes, Isabela S. Abordagem sobre vocabulários controlados para arquivos: conceitos, aplicações e metodologias. // Rodríguez Muñoz, J. V. et al. (Orgs.). Organización del conocimiento: sistemas de información abiertos: actas del XII Congreso ISKO España y II Congreso ISKO España y Portugal. Murcia: Universidad de Murcia, 2015.

Pavel, Silvia; Nolet, Diane (2002). Manual de terminologia. Canadá: Departamento de Tradução do Governo Canadense, 2002.

Scornik, Carolina; Sosa, Osvaldo (2007). Análisis de software libre para la gestión de lenguajes documentales: caso de estudio: software TemaTres. Corrientes: Universidad nacional del Nordeste.

The World Wide Web Consortium (W3C). 2012. Introduction to SKOS. http://www.w3.org/2004/02/skos/intro. (201603-16).

Troitiño, Sonia (coord.) (2016). Plano de classificação e tabela de temporalidade de documentos da UNESP: atividades meio. São Paulo: Cultura Acadêmica, 2016.

Unesco (1993). Diretrizes para o estabelecimento e desenvolvimento de tesauros monolíngües. [2. ed. rev. por Derek Austin e Peter Dale]; Trad. de Bianca Amaro de Melo; rev. de Lígia Maria Café de Miranda. Brasília: IBICT; SENAI.

Enviado: 2016-04-29. Segunda versión: 2016-06-13. Aceptado: 2016-06-04. 